\title{
АНАЛИТИЧЕСКАЯ ОЦЕНКА ДИНАМИКИ ПОТЕНЦИАЛА ВЛАЖНОСТИ В ЗОНЕ АЭРАЦИИ В ОРОШАЕМЫХ ЗЕМЛЯХ
}

\author{
Г.М. Егиазарян д.с.-х.н., А.Х. Хачатрян д.ф.-м.н., Р.А. Даниелян \\ Национальный аграрный университет Армении \\ yeghiazaryangurgen@gmail.com, agavard59@mail.ru, razmikdanielyan@mail.ru
}

\section{СВЕДЕНИЯ}

\section{Ключевые слова:}

капиллярный адсорбционный потенциал,

влагопроводность, уравнение влагопереноса, краевые и начальные условия, ряд Фурье.

\begin{abstract}
АННОТАЦИЯ
Рассматривается задача прогнозирования потенциала почвенной влаги с применением дифференциального уравнения влагопереноса в зоне аэрации. Предлагается аналитический подход вычисления потенциала влажности по глубине активного слоя почвы в период орошения. Полученный результат можно применить для различных типов почв с различными физико-химическими и гидрофизическими характеристиками. Для наглядной демонстрации предлагаемого подхода в конце работы приводятся численные результаты некоторых расчетов.
\end{abstract}

\section{Введение}

Оптимизация мелиоративного режима орошаемого земледелия заключается не только в увеличении урожайности сельскохозяйственных культур, но и в сохранении и улучшении плодородия почвы при условии оптимального управления водным режимом В зоне аэрации. В современных условиях, когда приходится всесторонне исследовать влияние физико-химических и гидрофизических параметров на водный режим в зоне «грунтовая вода - растение - почва - воздух», необходимо пользоваться методом математического моделирования в виде одномерного дифференциального уравнения математической фризики. В этом уравнении искомой функцией является полный потенциал влажности почвы, который определяется из следующего уравнения:

$$
H=\varphi-x \text {, }
$$

где $H$ - полный потенциал почвенной влаги, $\varphi$ капиллярный адсорбционный потенциал, зависящий от влажности почвы, $x$ - вертикальная координата с положительным направлением от поверхности вниз.

Всестороннему исследованию развития модели влагообмена в системе пахотного слоя почвы при наличии корневой системы посвящены многочисленные работы (С.Ф. Аверьянов, Л.М. Рекс, 1965, И.П. Айдаров, 1985, Г.М. Егиазарян, 2006).

Одномерная модель влагообмена записывается в следующем виде (С.Ф. Аверьянов, Л.М. Рекс, 1965, И.П. Айдаров, 1985, Г.М. Егиазарян, 2006):

$$
\frac{\partial \omega}{\partial \varphi} \frac{\partial H}{\partial t}=\frac{\partial}{\partial x}\left(K \frac{\partial H}{\partial x}\right)-e_{E T}
$$

где $\frac{\partial \omega}{\partial \varphi}$ - диффреренциальная влагоемкость, $K$ коэффициент влагопроводности почвы, зависящий от потенциала влажности, $e_{E T}$ - интенсивность поглощения влаги корнями растений.

Функциональные зависимости $K(\omega(\varphi))$ и $\varphi(\omega)$ для каждого типа почвы определяются индивидуально 
экспериментальным путем. В частности (И.П. Айдаров, 1985, Г.М. Егиазарян, 2006) предлагаются следующие зависимости:

$$
K=K_{f}\left[\frac{\omega-\omega_{0}}{m-\omega_{0}}\right]^{3.5}, \quad \varphi=\varphi_{0} \operatorname{tg}\left[\frac{\pi}{4}\left(\frac{\omega-\omega_{0}}{m-\omega_{0}}\right)\right],
$$

где $K_{f}$ - коэффициент фильтрации, $\omega$ - переменная влажность, $\omega_{0}$ - максимальная гигроскопичность почвы, $m$-пористость почвы, $\varphi_{0}$-капиллярный адсорбционный потенциал при влажности, гигроскопичности.

\section{Материал и методы}

Для решения уравнения (2) необходимо задать граничное и начальное условия на профиле почвы. В качестве начального условия задается распределение влажности или потенциала по глубине активного слоя почвы:

$$
H(x, 0)=\varphi(x, 0)-x=q(x)-x,
$$

где $q(x)$ - влажность в начальный момент времени.

Граничное условие на верхней границе имеет вид

$$
-\left.\frac{\partial H}{\partial x}\right|_{x=0}=1-q_{0} e^{-a t}
$$

где $q_{0}$ - максимальная скорость впитывания, $a$ параметр обратного времени, характеризующий темп впитывания в почву. Для установления оптимального водного режима и регулирования уровня грунтовых вод на нижней границе при наличии дренажа граничное условие принимает следующий вид:

$$
-\left.K \frac{\partial H}{\partial x}\right|_{x=l}=D\left(h_{D}-h_{g}\right)
$$

где $D$ - модуль дренажного стока, $h_{d}$ - глубина расположения дренажа, $h_{\mathrm{g}}$ - глубина грунтовых вод.

Из уравнения (3) имеем

$$
\omega(\varphi)=\omega_{0}+\frac{4\left(m-\omega_{0}\right)}{\pi} \operatorname{arctg} \frac{\varphi}{\varphi_{0}} .
$$

Разлагая уравнение (5) в ряд Тейлора в окрестности $\varphi_{0}$ и ограничиваясь линейным членом разложения, получим

$$
\omega(\varphi)=m+\frac{2\left(m-\omega_{0}\right)}{\pi \varphi_{0}}\left(\varphi-\varphi_{0}\right)
$$

$$
\frac{\partial \omega}{\partial \varphi}=\frac{\left(m-\omega_{0}\right)}{\pi \varphi_{0}}=\theta_{0} .
$$

В качестве коэффициента влагопроводности почвы берем его усредненное значение:

$$
K(\omega)=K_{0}=\text { cosnt } .
$$

Предположим, что интенсивность поглощения влаги корнями растений постоянна $\left(e_{E T}=e_{0}=\right.$ const $)$. Результаты можно легко обобщить, когда $e_{E T}$ зависит от времени и координат.

Окончательно уравнение (2) относительно искомой функции примет вид

$$
\frac{\partial \omega}{\partial t}=a^{2} \frac{\partial^{2} \varphi}{\partial x^{2}}-a_{1}
$$

где введены следующие обозначения:

$$
a^{2}=\frac{K_{0}}{\theta_{0}}, \quad a_{1}=\frac{e_{0}}{\theta_{0}} .
$$

Теперь к уравнению (7) присоединим граничное и начальное условия

$$
\begin{gathered}
\left.\frac{\partial \varphi}{\partial t}\right|_{x=0}=q_{1(t)},\left.\quad \frac{\partial \varphi}{\partial t}\right|_{x=l}=q_{2(t)}, \\
\varphi(x, 0)=q(x) .
\end{gathered}
$$

Здесь

$$
\begin{gathered}
q_{1}(t)=q_{0} e^{-\alpha t}, \\
q_{2}(t)=-\frac{D\left(h_{D}-h_{g}\right)}{K_{f}}+1,
\end{gathered}
$$

где $q_{1}(t)$ - скорость водообмена над поверхностью почвы, а $q_{2}(t)$ - скорость влагообмена на глубине почвы.

Водный режим, являющийся основным фрактором управления воздушным, тепловым, химическим и биологическим режимами почвы, характеризируется влажностью (потенциала) и глубиной грунтовых вод. Именно этими параметрами определяется почвенномелиоративное состояние орошаемых почв в разных гидрогеологических и климатических условиях Араратской долины.

Начально-краевая задача (7-9) отличается от классической задачи (А.Н. Тихонов, А.А. Самарский, 1977, Н.С. Пискунов, 1970) тем, что здесь граничные условия (8) накладываются не на искомую функцию $\varphi$, а на ее производные. 


\section{Результаты и анализ}

Введем новую функцию посредством

$$
U(x, t)=\varphi(x, t)-A(t) x^{2}-B(t) x,
$$

где $A(t)$ и $B(t)$ - неизвестные функции и подлежат определению. Эти функции выбираем так, чтобы функция $U(x, t)$ удовлетворяла нулевым граничным условиям.

$$
\left.\frac{\partial U}{\partial x}\right|_{x=0}=\left.\frac{\partial U}{\partial x}\right|_{x=l}=0
$$

Имеем

$$
\left.\frac{\partial U}{\partial x}\right|_{x=0}=\left.\frac{\partial \varphi}{\partial x}\right|_{x=0}-B(t)=q_{1(t)}-B(t) .
$$

Отсюда следует, что

$$
\begin{gathered}
B(t)=q_{1}(t), \\
\left.\frac{\partial U}{\partial x}\right|_{x=l}=\left.\frac{\partial \varphi}{\partial x}\right|_{x=l}-2 A(t) l-B(t)= \\
=q_{2}(t)-2 A(t) l-B(t)=0
\end{gathered}
$$

или

$$
A(t)=\frac{q_{2}(t)-q_{1}(t)}{2 l}
$$

Итак,

$$
U(x, t)=\varphi(x, t)-\frac{q_{2}(t)-q_{1}(t)}{2 l} x^{2}-q_{1}(t) x .
$$

Теперь рассмотрим начальное условие (9).

Из $\varphi(x, 0)=q(x)$ следует, что

$$
\begin{aligned}
U(x, 0)= & q(x)-\frac{q_{2}(0)-q_{1}(0)}{2 l} x^{2}- \\
& -q_{1}(0) x \equiv \psi(x) .
\end{aligned}
$$

Таким образом, относительно функции $U(x, t)$ мы приходим к следующей начально-краевой задаче:

$$
\begin{aligned}
& \frac{\partial U}{\partial t}=a^{2} \frac{\partial^{2} U}{\partial x^{2}}=f(x, t) \\
& \left.\frac{\partial U}{\partial x}\right|_{x=0}=\left.\frac{\partial U}{\partial x}\right|_{x=l}=0 \\
& U(x, 0)=\Psi(x)
\end{aligned}
$$

где $f(x, t)=2 a^{2} A(t)-a_{1}-A^{\prime}(t) x^{2}-B^{\prime}(t) x$.
Заметим, что если $W(x, t)$ и $V(x, t)$ являются решениями граничных задач

$$
\begin{aligned}
& \left\{\begin{array}{l}
\frac{\partial W}{\partial t}=a^{2} \frac{\partial^{2} W}{\partial x^{2}}+f(x, t) \\
\left.\frac{\partial W}{\partial x}\right|_{x=0}=\left.\frac{\partial W}{\partial x}\right|_{x=l}=0 \\
W(x, 0)=0
\end{array}\right. \\
& \left\{\begin{array}{l}
\frac{\partial V}{\partial t}=a^{2} \frac{\partial^{2} V}{\partial x^{2}} \\
\left.\frac{\partial V}{\partial x}\right|_{x=0}=\left.\frac{\partial V}{\partial x}\right|_{x=l}=0, \\
V(x, 0)=\psi(x)
\end{array}\right.
\end{aligned}
$$

то в силу линейности задачи (18)-(20) ее решение можно представить в виде

$$
U(x, t)=W(x, t)+V(x, t) .
$$

Займемся решением граничной задачи (21). Ее решение ищем в виде

$$
W_{n}(x, t)=X_{n}(x) T_{n}(t),
$$

$$
X_{n}(x)=\cos \frac{\pi n}{l} x, \quad f_{n}(x, t)=X_{n}(x) F_{n}(t) .
$$

Учитывая, что $W(x, 0)=0$ для фрункции $T_{n}(t)$, получаем следующее обыкновенное дифференциальное уравнение:

$$
T_{n}^{\prime}(t)=-\left(\frac{a \pi n}{l}\right)^{2} T_{n}(t)+F_{n}(t)
$$

с начальным условием

$$
T_{n}(0)=0 .
$$

Решение начальной задачи (25), (26) имеет вид

$$
T_{n}(t)=\int_{0}^{t} e^{-\left(\frac{a \pi n}{l}\right)^{2}(t-\tau)} F_{n}(\tau) d \tau
$$

Теперь функцию $f(x, t)$ продолжаем четно по аргументу $x$ на интервале $[-l, 0]$. Поскольку

$$
f_{n}(x, t)=X_{n}(x) F_{n}(t)=\cos \frac{\pi n}{l} x \cdot F_{n}(t)
$$

то в качестве коэффрициентов Фурье будут

$$
\begin{aligned}
F_{n}(t) & =\frac{1}{l} \int_{-l}^{l} f(\xi, t) \cos \frac{\pi n}{l} \xi d \xi= \\
& =\frac{2}{l} \int_{0}^{l} f(\xi, t) \cos \frac{\pi n}{l} \xi d \xi .
\end{aligned}
$$


Подставляя выражение для $f(x, t)$ из (20), получим

$$
F_{n}(t)=\frac{2}{l} \int_{0}^{l}\left[\begin{array}{l}
2 a^{2} A(t)-a_{1}- \\
-A^{\prime}(t) \xi^{2}-B^{\prime}(t) \xi
\end{array}\right] \cos \frac{\pi n}{l} \xi d \xi .
$$

Итак, решение задачи (21) записывается в виде

$$
\begin{aligned}
& W(x, t)=\sum_{n=0}^{\infty} X_{n}(x) T_{n}(t)= \\
& =\sum_{n=0}^{\infty}\left(\int_{0}^{t} e^{-\left(\frac{a \pi n}{l}\right)^{2}(t-\tau)}\left[\frac{2}{l} \int_{0}^{l} f(\xi, t) \cos \frac{\pi n}{l}(\xi d \xi)\right] d \tau\right) \cos \frac{\pi n}{l} x
\end{aligned}
$$

Теперь переходим к задаче (22). Здесь решение также ищем в виде

$$
V(x, t)=X(x) T(t)
$$

Учитывая граничные условия из (22), будем иметь

$$
\begin{aligned}
& X^{\prime \prime}(x)+\lambda X=0, \\
& X^{\prime}(0)=X^{\prime}(l)=0 .
\end{aligned}
$$

Задача (33), (34) представляет собой задачу ШтурмаЛиувилля, решение которой допускает вид

$$
X_{n}(x)=C_{n} \cos \frac{\pi n}{l} x, \quad \lambda=\lambda_{n}=\left(\frac{\pi n}{l}\right)^{2} .
$$

Решение уравнения

$$
T^{\prime}(t)+\left(\frac{a \pi n}{l}\right)^{2} T(t)=0
$$

можно записать в виде

$$
T^{\prime}(t)=D_{n} e^{-\left(\frac{a \pi n}{l}\right)^{2}} .
$$

Тогда общее решение задачи (22) представим в виде ряда

$V(x, t)=\sum_{n=0}^{\infty} V_{n}(x, t)=\sum_{n=0}^{\infty} C_{n} \cos \frac{\pi n}{l} x e^{-\left(\frac{a \pi n}{l}\right)^{2} t}$,

где коэфффициенты $C_{n}$ подлежат определению. Из начального условия $V(x, 0)=\Psi(x)$ получаем

$$
\psi(x)=C_{n} \cos \frac{\pi n}{l} x
$$

где

$$
C_{n}=\frac{1}{l} \int_{-l}^{l} \psi(\xi) \cos \frac{\pi n}{l} \xi d \xi, n=0,1,2,3 \ldots
$$

Функцию $\Psi$ также продолжаем четно на интервале $\{-l, 0]$. Имеем

$$
C_{n}=\frac{2}{l} \int_{0}^{l} \psi(\xi) \cos \frac{\pi n}{l} \xi d \xi
$$

Таким образом,

$$
\begin{aligned}
& C_{n}=\frac{2}{l} \int_{0}^{l}\left[q(\xi)-\frac{\left[q_{2}(0)-q_{1}(0)\right]}{2 l} \xi^{2}-q_{1}(0) \xi\right] \\
& \cdot \cos \frac{\pi n}{l} \xi d \xi, \quad n=0,1,2,3 \ldots
\end{aligned}
$$

Следовательно,

$$
\begin{aligned}
& U(x, t)=\sum_{n=0}^{\infty} C_{n} \cos \frac{\pi n}{l} x e^{-\left(\frac{a \pi n}{l}\right)^{2}}+ \\
& +\sum_{n=0}^{\infty}\left(\int_{0}^{t} e^{-\left(\frac{a \pi n}{l}\right)^{2}(t-\tau)}\left[\frac{2}{l} \int_{0}^{l} f(\xi, \tau) \cos \frac{\pi n}{l} \xi d \xi\right] d \tau\right) \cos \frac{\pi n}{l} x
\end{aligned}
$$

Окончательное решение искомой задачи (8)-(9) примет вид

$$
\varphi(x, t)=U(x, t)+A(t) x^{2}+B(t) x
$$

где функции $A(t)$ и $B(t)$ задаются посредством уравнений (14), (15), а $U(x, t)$ согласно уравнению (43).

Рассмотрим следующий частный случай. В качестве и берем их усредненные значения.

$$
q_{1}=\bar{q}_{1}=\text { const }, \quad q_{2}=\bar{q}_{2}=\text { const }
$$

Тогда имеем

$$
f(x, t)=\frac{a^{2}\left(\bar{q}_{2}-\bar{q}_{1}\right)}{l}-a_{1}=\text { const } .
$$

Нетрудно убедиться, чти решение неоднородной задачи (21) примет вид

$$
W(x, t)=2\left(\frac{a^{2}\left(\bar{q}_{2}-\bar{q}_{1}\right)}{l}-a_{1}\right) t .
$$

Теперь рассмотрим соответствующее однородное уравнение (22)

Сперва вычислим коэффрициенты Фурье $C_{0}$ и $C_{n}$, предполагая, что в начальный момент времени влажность допускает следующее представление $q(x)=l^{2}-x^{2}$, $0 \leq x \leq l$. 
Имеем

$C_{n}=\frac{2}{l} \int_{0}^{l} \psi(x) \cos \frac{\pi n}{l} x d x=$

$=2 l \int_{0}^{l} \cos \frac{\pi n}{l} x d x-\frac{2}{l} \int_{0}^{l} x^{2} \cos \frac{\pi n}{l} x d x-\frac{\left(\bar{q}_{2}-\bar{q}_{1}\right)}{l^{2}} \int_{0}^{l} x^{2} \cos \frac{\pi n}{l} x d x-$

$-\frac{2 \bar{q}_{1}}{l} \int_{0}^{l} \cos \frac{\pi n}{l} x d x, \quad n=0,1,2,3, \ldots$

Очевидно, что первый интеграл обращается в нуль. Используя значение интегралов

$\int_{0}^{l} x \cos \frac{\pi n}{l} x d x=\left\{\begin{array}{ll}-\frac{2 l^{2}}{\pi^{2} n^{2}}, & \text { если } n=2 k+1, \\ 0, & \text { если } n=2 k\end{array} \quad n \neq 0\right.$,

$\int_{0}^{l} x^{2} \cos \frac{\pi n}{l} x d x=\left\{\begin{array}{l}-\frac{2 l^{3}}{\pi^{2} n^{2}}, \text { если } n=2 k+1, \\ \frac{2 l^{3}}{\pi^{2} n^{2}}, \text { если } n=2 k\end{array} \quad n \neq 0\right.$,

будем иметь

$C_{n}= \begin{cases}-\frac{2 l\left(\bar{q}_{2}-\bar{q}_{1}\right)}{\pi^{2} n^{2}}-\frac{4 l^{2}}{\pi^{2} n^{2}}, & n \neq 0, \\ \frac{4 l^{2}}{\pi^{2} n^{2}}+\frac{4 l q_{1}}{\pi^{2} n^{2}}+\frac{2 l\left(\bar{q}_{2}-\bar{q}_{1}\right)}{\pi^{2} n^{2}}, & \text { если } n=2 k\end{cases}$

$C_{0}=\frac{4}{3} l^{2}-\frac{1}{3}\left(\bar{q}_{2}-\bar{q}_{1}\right) l-\bar{q}_{1} l, \quad n=0$.

Итак, решение исследуемой нами начально-краевой задачи в этом случае приобретает вид

$$
\begin{aligned}
& \varphi(x, t)=\frac{\left(\bar{q}_{2}-\bar{q}_{1}\right) x^{2}}{2 l}+\bar{q}_{1} x+\frac{C_{0}}{2}+ \\
& +\sum_{n=1}^{\infty} C_{n} \cos \frac{\pi n}{l} x e^{-\left(\frac{a \pi n}{l}\right)^{2} t}+2\left(\frac{a^{2}\left(\bar{q}_{2}-\bar{q}_{1}\right)}{l}-a_{1}\right) t
\end{aligned}
$$

где $C_{n}$ задаются посредством уравнения (51). Заметим, что соответствующий ряд сходится равномерно, поскольку для него мажорантой служит сходящийся числовой ряд вида

$$
c \cdot \sum_{n=1}^{\infty} \frac{1}{n^{2}} \quad(c=\text { const }) .
$$

Ниже для демонстрации развитого нами подхода приведем результаты некоторых численных расчетов. Пусть глубина почвы $l=2 \quad \mu$ скорость влагообмена на глубине $\bar{q}_{2}=-1,5$ м/сут и $\bar{q}_{1}=-0,05$ м/сут на поверхности почвы грунта равны соответственно, $a_{1}=0,05 \mathrm{~m} /$ сут, $a=0,5 \mathrm{~m} /$ сут. На рисунке 1. изображена зависимость капиллярно-адсорбционного потенциала почвы $\varphi(x, t)$ от глубины $0 \leq x \leq 2$ почвы за межполивной период влагообмена.

На рисунке 2 отображена аналитическое значение потенциала влажности в виде графрика фрункции с двумя переменными.

Численные расчеты выполнены на ЭВМ. Код написан на языке Python.

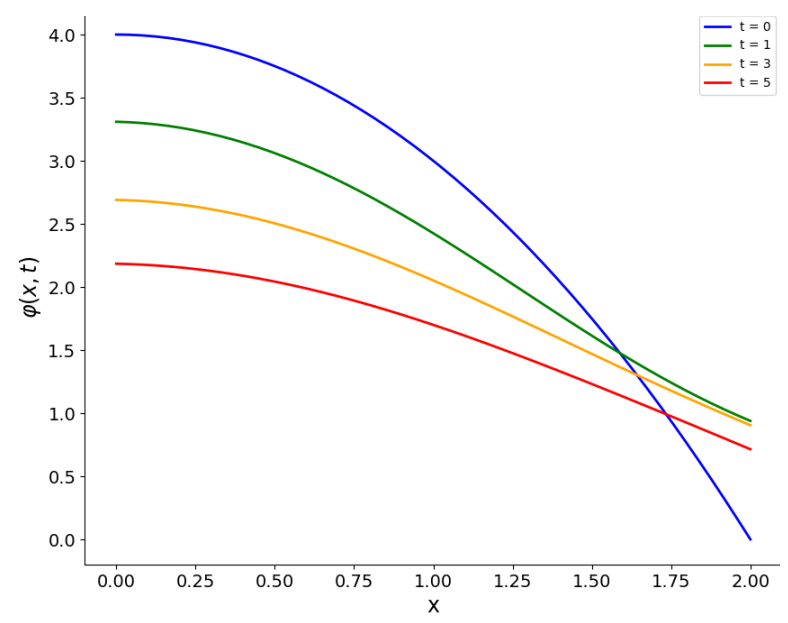

Рис. 1. Динамика капиллярно-адсорбционного потенциала в межполивной период орошения за первые, третьи и пятые сутки влагообмена.

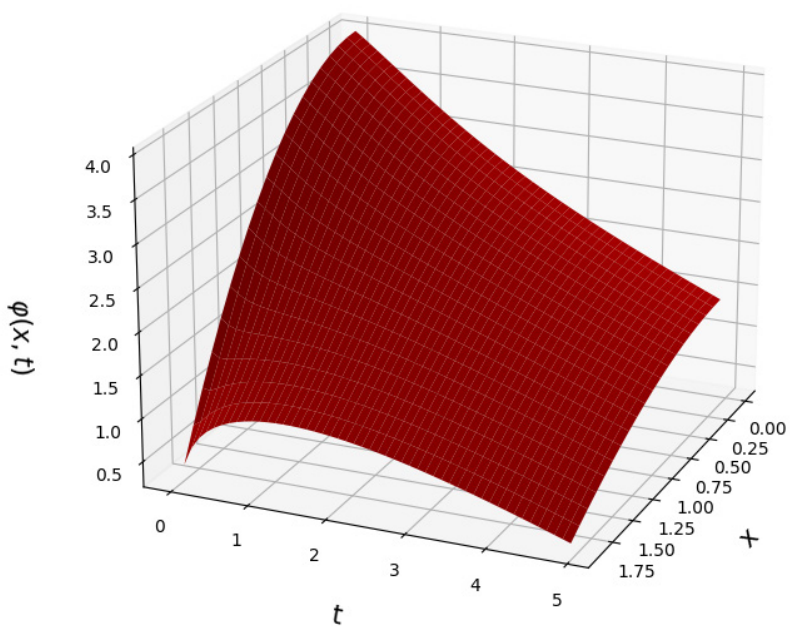

Рис. 2. Двухмерное распределение потенциала влажности активного слоя почвы по глубине и по времени. 


\section{Заключение}

Результаты численного расчета и их сопоставление с полевыми данными показывают, что в период орошения предлагаемый аналитический подход вычисления потенциала влажности активного слоя почвы по глубине с достаточной точностью выявляет динамику капиллярно-адсорбционного потенциала и позволяет применять его для разных типов почв с различными физико-химическими и гидрофизическими характеристиками.

Замечание. Созданию пакета программ по определению потенциала почвенной влаги для конкретных типов почв с конкретными физико-химическими параметрами предполагается посвятить отдельную работу.

\section{Литература}

1. Аверьянов С.Ф., Рекс Л.М. Некоторые вопросы предупреждения засоления орошаемых земель и меры борьбы с ним в Европейской части СССР. - М.: Колос, 1965. - С. 90-149.

2. Айдаров И.П. Регулирование водно-солевого и питательного режимов орошаемых земель. - М: Агропромиздат, 1985.

3. Егиазарян Г.М. Прогнозирование и регулирование водно-солевого режима в орошаемых землях. - Ер.: ГАУА, 2006.

4. Тихонов А.Н., Самарский А.А. Уравнения математической физики. - М.: Наука, 1977.

5. Пискунов Н.С. Дифференциальное и интегральное исчисление. - Т. 2. - М.: Наука, 1970.

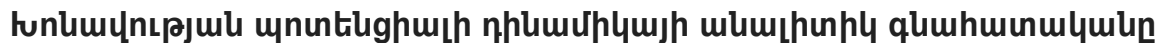 nnnqtih hnntinh uthugghn qnunnul}

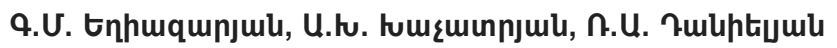

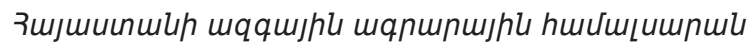

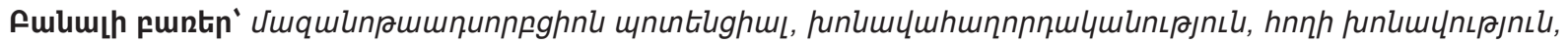

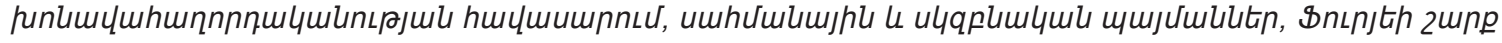

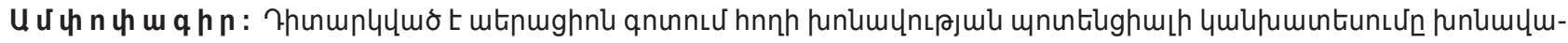

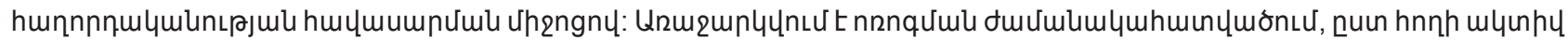

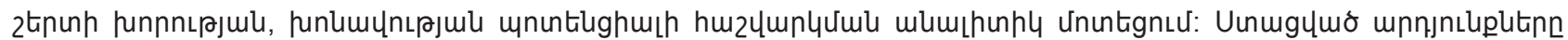

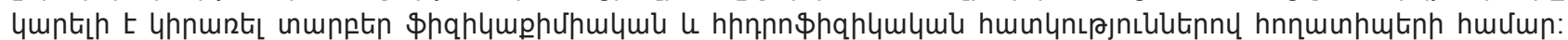

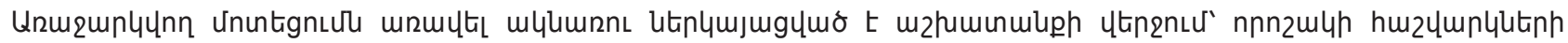

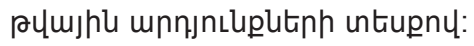

\section{Analytic Assessment of the Moisture Capacity Dynamics in the Aeration Zone of Irrigated Lands}

\section{G.M. Yeghiazaryan, A.Kh. Khachatryan, R.A. Danielyan}

Armenian National Agrarian University

Keywords: capillary adsorption potential, moisture conductivity, moisture transfer equation, boundary and initial conditions, Fourier series

A b s t r a c t . The issue of forecasting soil moisture capacity using differential equation of moisture transfer in the aeration zone has been considered. An analytical approach for estimating moisture capacity per the depth of active soil layer in the irrigation period has been recommended. The obtained outcomes can be used for different soil types with various physicochemical and hydrophysical properties. To make the recommended approach more demonstrative the results of some numerical computations are introduced at the end of the current work. 